\title{
Diagnosis of gestational diabetes mellitus: comparison between National Diabetes Data Group and Carpenter- Coustan criteria
}

\author{
Dittakarn Boriboonhirunsarn, Tripop Lertbunnaphong, Kamaitorn Tientong \\ Department of Obstetrics and Gynaecology, Faculty of Medicine, Siriraj Hospital, Mahidol University, \\ Bangkok 10700, Thailand
}

\begin{abstract}
Background: A 100-g oral glucose tolerance test (OGTT) is commonly used to diagnose gestational diabetes mellitus (GDM). Carpenter-Coustan (CC) criteria, based on lower threshold plasma glucose values than the National Diabetes Data Group (NDDG) criteria, result in an apparently increased prevalence of GDM. However, the extent of the increase is not known, and effects on perinatal outcome are not clear.

Objective: To evaluate the increase in the prevalence of GDM if CC criteria are applied to OGTT results, we compared findings with NDDG criteria. Pregnancy outcomes between women without GDM and those with GDM diagnosed by NDDG and CC criteria were compared.

Methods: A total of 640 at-risk pregnant women were studied. They were either diagnosed as having GDM by initial testing ( 145 women), or repeat testing at 24-28 weeks of gestation (495 women). CC criteria were applied to the OGTT results and prevalence of GDM was re-evaluated.

Results: The apparent prevalence of GDM increased by $22.2 \%$ using CC criteria. The change was $27.6 \%$ at the initial test and 31.5\% at repeat tests during 24-28 weeks of gestation. Infant birth weight in GDM diagnosed by either NDDG or CC criteria was significantly higher than in the negative OGTT group $(P<0.001)$. Rates of macrosomia were comparable. Neonatal hypoglycemia was $14.6 \%$ in the NDDG group, $8.2 \%$ in CC only group, and $4.6 \%$ in negative OGTT group $(P<0.001)$.

Conclusion: The CC criteria identify 22\% more cases of GDM than NDDG criteria during initial and repeat tests.
\end{abstract}

Keywords: Carpenter-Coustan, gestational diabetes mellitus, NDDG, prevalence

Gestational diabetes mellitus (GDM) is defined as carbohydrate intolerance of variable severity with onset or first recognition during pregnancy [1-3]. GDM is associated with an increased incidence of maternal and perinatal morbidity and higher risk for obesity and diabetes later in life. To date, recommendations for screening and diagnosis of GDM still vary among expert committees and there are no consensus conclusions regarding appropriate screening and diagnostic approaches [1, 3, 4].

A 100-gram oral glucose tolerance test (OGTT) is a common method for diagnosis of GDM, as recommended by the American Congress of Obstetricians and Gynecologists (ACOG) [3]. However, 2 different sets of thresholds have been

Correspondence to: Dittakarn Boriboonhirunsarn, Department of Obstetrics and Gynecology, Faculty of Medicine, Siriraj Hospital, Mahidol University, Bangkok 10700, Thailand. E-mail:sidbr@mahidol.ac.th proposed and are used widely for GDM diagnosis. For fasting plasma glucose, and at 1, 2, and 3 hours after a 100-g glucose load, the National Diabetes Data Group (NDDG) proposed cut-off levels of 105, 190, 165, and $145 \mathrm{mg} / \mathrm{dL}$, while Carpenter and Coustan proposed a lower cut-off values of 95, 180, 155, and $140 \mathrm{mg} / \mathrm{dL}$, respectively, as diagnostic thresholds. For both sets of criteria, a positive diagnosis requires that 2 or more test thresholds be met or exceeded [5, 6].

The Carpenter-Coustan (CC) criteria, based on lower threshold plasma glucose values, will inevitably result in an increased apparent prevalence of GDM. However, the magnitude of this increase across populations with different risks of carbohydrate intolerance is not exactly known. In addition, effects on perinatal outcome are even less clear [7-12].

The aim of this study was to evaluate magnitude of changes in the prevalence of GDM if CC criteria were applied rather than NDDG criteria. The changes were evaluated for initial testing during the first 
antenatal visit and the repeat testing during 24-28 weeks of gestation. In addition, pregnancy outcomes were also evaluated and compared between those without GDM, GDM diagnosed by NDDG criteria, and GDM diagnosed by CC criteria alone.

\section{Methods}

This retrospective study was approved by the Siriraj Institutional Review Board (SIRB, Si339/2010). According to our institutional guidelines, like those for most hospitals in Thailand, a risk factor-based selective screening approach was used to diagnose GDM. This consists of a 50-g glucose challenge test (GCT) as a screening test with a cut off level of 140 $\mathrm{mg} / \mathrm{dL}$, and 100-g OGTT as a confirmatory test and using NDDG criteria. The process is offered during the first antenatal visit and repeated during 24-28 weeks of gestation if the initial tests were normal [13].

A total of 640 consecutive cases of at-risk pregnant women who were either diagnosed with GDM by initial testing during their first antenatal visit before 20 weeks of gestation (145 women), or received a 100-g OGTT during both first and repeat testing during 24-28 weeks of gestation (495 women), were included in this study. Exclusion criteria were pregestational diabetes mellitus, metabolic syndrome, multiple gestation, or fetal anomalies.

Data from all OGTT results were retrieved from medical records. The CC criteria were then applied to all OGTT results from the pregnant women and prevalence of GDM was re-evaluated and compared with those diagnosed using NDDG criteria. The results were grouped into: GDM diagnosed by both NDDG and CC criteria (NDDG), GDM not diagnosed by either NDDG and CC criteria (negative OGTT), and GDM diagnosed by CC criteria alone (CC only). The results from initial and repeat tests were paired and the prevalence of GDM was reevaluated using CC criteria.

In addition, maternal and neonatal outcomes were evaluated and compared between the groups. These outcomes included gestational age at delivery, mode of delivery, infant birth weight, neonatal hypoglycemia, birth asphyxia, and NICU admission.

Descriptive statistics including mean, standard deviation (SD), number, and percentage are used to describe various baseline characteristics as appropriate. A chi-square test and analysis of variance (ANOVA) were used to compare various variables between groups as appropriate. $P<0.05$ was considered statistical significant.

\section{Results}

Of 640 pregnant women that were included in this study, 145 women (22.6\%) were diagnosed with GDM during their first visit. Another 495 women received repeat 100-g OGTT during 24-28 weeks of gestation. Baseline characteristics are shown in Table 1. Mean age was $32.5 \pm 5.1$ years, mean prepregnancy body mass index was $23.6 \pm 4.6 \mathrm{~kg} / \mathrm{m}^{2}$, and $43.2 \%$ were nulliparous. The 3 most common risks for GDM were age $\geq 30$ years (73.6\%), family history of DM (45.6\%), and obesity (22.3\%).

Table 1. Baseline characteristics of the study population $(N=640)$

\begin{tabular}{ll}
\hline Characteristics & Mean \pm SD \\
\hline Age (year) & $32.5 \pm 5.1$ \\
Prepregnancy body mass index $\left(\mathrm{kg} / \mathrm{m}^{2}\right)$ & $23.6 \pm 4.6$ \\
Total weight gain $(\mathrm{kg})$ & $12.9 \pm 4.4$ \\
\hline & $\mathbf{n ( \% )}$ \\
\hline Parity & $277(43.2 \%)$ \\
0 & $279(43.6 \%)$ \\
1 & $84(13.1 \%)$ \\
$\geq 2$ & \\
Gestational diabetes mellitus $(\mathrm{GDM})$ risks* & $471(73.6)$ \\
Age $\geq 30$ years & $292(45.6)$ \\
Family history of DM & $143(22.3)$ \\
$\quad$ Prepregnancy obesity & $15(2.3)$ \\
History of macrosomia infant & $15(2.3)$ \\
Hypertension & $5(0.8)$ \\
Previous history of GDM & $2(0.3)$ \\
History of fetal anomalies & $2(0.3)$ \\
History of stillbirth & \\
\hline
\end{tabular}

*Risks according to institutional guidelines [13] 
Paired results from the first and second OGTT when both NDDG and CC criteria were applied are indicated in Table 2. Overall, GDM was diagnosed in 275 patients (43\%), 145 cases during first visit and another 130 cases during repeat testing. If CC criteria were applied, 61 additional patients would be diagnosed with GDM, i.e., 22.2\% increase in overall prevalence. These patients would have been missed and received no treatment according to the current guidelines.

We note that 40 more women would have been diagnosed on their first visit if CC criteria were applied, i.e., $27.6 \%$ increase in rate of diagnosis. The diagnosis could have been made a mean of 13 weeks earlier than with using NDDG criteria. During the repeat testing, 130 women (26.3\%) were diagnosed with GDM using NDDG criteria and another 41 women would have been diagnosed with CC criteria, i.e., $31.5 \%$ increase in rate of diagnosis.
Comparisons of pregnancy outcomes were made between 3 groups, i.e., those without GDM by both criteria (negative OGTT, 304 cases), those with GDM by either NDDG or CC criteria (NDDG, 275 cases), and those with GDM by CC criteria only (CC only, 61 cases) as shown in Table 3. No significant difference was found between groups with regard to gestational age at delivery, cesarean delivery, macrosomia, neonatal ICU admission, or birth asphyxia. While infant birth weight was comparable between women in the NDDG and CC only groups, it was significantly lower in the negative OGTT group. Neonatal hypoglycemia was also significantly different between groups and significant trend was observed, i.e., $14.6 \%$ in the NDDG group, $8.2 \%$ in the CC only group, and $4.6 \%$ in the negative OGTT group $(P<$ 0.001).

Table 2. Diagnosis of gestational diabetes mellitus (GDM) using National Diabetes Data Group (NDDG) and Carpenter-Coustan (CC) criteria $(N=640)$

\begin{tabular}{lcccc}
\hline \multirow{2}{*}{ First OGTT result } & \multicolumn{4}{c}{ Second OGTT result } \\
\cline { 2 - 5 } & N/A & Negative OGTT & CC only & NDDG \\
\hline NDDG & 145 & - & - & - \\
Negative OGTT & - & 304 & $32^{\mathrm{a}}$ & 119 \\
CC only & - & $20^{\mathrm{a}, \mathrm{b}}$ & $9^{\mathrm{a}, \mathrm{b}}$ & $11^{\mathrm{b}}$ \\
\hline OGTT, 100-gram oral glucose tolerance test & & \\
a Cases of GDM that would be diagnosed if CC criteria were applied (61 cases) & \\
${ }^{\mathrm{b}}$ Cases of GDM that would have been diagnosed since their first visit if CC criteria were applied (40 cases)
\end{tabular}

Table 3. Perinatal outcomes of the study population $(N=640)$

\begin{tabular}{lllll}
\hline & $\begin{array}{c}\text { Negative OGTT } \\
\boldsymbol{n}=\mathbf{3 0 4}\end{array}$ & $\begin{array}{l}\text { NDDG } \\
\boldsymbol{n}=\mathbf{2 7 5}\end{array}$ & $\begin{array}{c}\text { CC only } \\
\boldsymbol{n}=\mathbf{6 1}\end{array}$ & $\mathbf{P}$ \\
\hline Gestational age (weeks of gestation) & $38.2 \pm 1.6$ & $38.2 \pm 1.4$ & $38.5 \pm 1.7$ & 0.352 \\
Cesarean delivery & $136(44.7 \%)$ & $148(53.8 \%)$ & $32(52.5 \%)$ & 0.081 \\
Infant birth weight (g) & $3079.7 \pm 452 *$ & $3244.7 \pm 455.1$ & $3237.4 \pm 461.5$ & $<0.001$ \\
Macrosomia & $11(3.6 \%)$ & $15(5.4 \%)$ & $3(4.9 \%)$ & 0.563 \\
Neonatal hypoglycemia & $14(4.6 \%)$ & $40(14.5 \%)$ & $5(8.2 \%)$ & $<0.001$ \\
Neonatal ICU admission & $1(0.3 \%)$ & $2(0.7 \%)$ & $1(1.6 \%)$ & 0.476 \\
Asphyxia & $14(4.6 \%)$ & $7(2.5 \%)$ & $4(6.6 \%)$ & 0.235 \\
\hline
\end{tabular}

OGTT, 100-gram oral glucose tolerance test; NDDG, National Diabetes Data Group; CC, Carpenter-Coustan *Significant difference from the other 2 groups by post hoc comparison, $P<0.05$ 


\section{Discussion}

Methods for screening and diagnosis of GDM are still controversial. A two-step approach consisting of screening with 50-g GCT, followed by 100-g OGTT is commonly recommended many authorities, including the American Congress of Obstetricians and Gynecologists (ACOG). However, for a 100-g OGTT, 2 diagnostic criteria have been proposed, i.e., NDDG and CC criteria, and advantages of each are still not clear [7-10, 12, 14].

Our results demonstrated that overall apparent prevalence of GDM increased by $22.2 \%$ if CC criteria were used for the diagnosis. The results also showed that CC criteria would identify $27.6 \%$ more GDM cases during their first visit, at an average of 13 weeks earlier than using NDDG criteria. These findings are similar to those of previous studies, which found an increase in GDM prevalence of between $30 \%$ and $50 \%[7,12]$. These findings can be explained by the increased sensitivity of CC criteria because of lower thresholds compared with NDDG criteria. Similar results were observed during repeat testing and a $31.5 \%$ increase in GDM diagnosis was observed. This has not previously been reported to our knowledge. According to current NDDG guidelines, these patients would have been missed and not have been counseled and treated.

Whether women with OGTT values above the CC threshold, but below the NDDG threshold, are at increased risk of adverse perinatal outcomes has been inconsistently reported $[8,9,11,12,14,15]$. The results of this study revealed that, while mean birth weight was comparable between CC only and NDDG groups, it was significantly greater than in those without GDM $(P<0.001)$. However, the difference is of minimal clinical importance and the rate of macrosomia was not significantly different between the 3 groups. A significantly increased trend was observed for neonatal hypoglycemia. Neonatal hypoglycemia was $4.6 \%$ in those without GDM, $8.2 \%$ in the CC only group, and $14.5 \%$ in the NDDG group. However, the rate in the CC only group and in those without GDM, might be underestimated because newborns of mothers without GDM by NDDG criteria were not routinely screened for hypoglycemia. Like this study, other studies have also found an increase in both maternal and neonatal adverse outcomes in GDM women diagnosed by CC criteria only. The risks included higher rates of cesarean and operative vaginal delivery $[8,9,11,12]$, macrosomia [8, 9, 11, 12, 14, 15], neonatal hyperbilirubinemia and hypoglycemia $[12,15]$.
Some limitations of the current study should be noted. The study population might not be representative, because those who were lost to follow up and did not receive repeat tests were not included. Thus, the increased rate of diagnosis as well as the rate of adverse outcomes might be less accurate than expected. However, baseline characteristics, such as GDM risks, did not significantly deviate from previous studies at the same institution $[16,17]$. In addition, according to guidelines, all at-risk women receive dietary counseling during their antenatal care, which might decrease their chances of GDM diagnosis by the second test as well as adverse perinatal outcomes. Our results might not be applicable to other populations because of varying risks for GDM and varying degrees of carbohydrate intolerance in other populations.

To determine whether the more inclusive CC criteria should be adopted and replace the NDDG criteria requires thorough considerations of many aspects, including work load and cost. Costeffectiveness analysis to quantify the risks and benefits of increased GDM diagnosis and related issues, especially impact on adverse perinatal outcomes, remains to be determined.

\section{Conclusion}

If CC criteria are used, the overall GDM prevalence would apparently increase by $22.2 \%$. The CC criteria would have identified $27.6 \%$ more GDM cases during their initial test and 31.5\% more during their repeat tests during 24-28 weeks of gestation. However, although an infant's birth weight and rate of neonatal hypoglycemia were significantly greater among those diagnosed by CC criteria than in the negative OGTT group, other perinatal outcomes were comparable.

\section{Acknowledgment}

The authors received no outside funding for this study and have no conflict of interest to report.

\section{References}

1. American Diabetes Association. Diagnosis and classi cation of diabetes mellitus (Position Statement). Diabetes Care. 2009; 32(Suppl 1):S62-7.

2. Expert Committee on the Diagnosis and Classi cation of Diabetes Mellitus. Report of the Expert Committee on the Diagnosis and Classification of Diabetes Mellitus. Diabetes Care. 1997; 20:1183-97.

3. The American College of Obstetricians and Gynecologists. Committee opinion number 504: 
Screening and diagnosis of gestational diabetes mellitus. Obstet Gynecol. 2011; 118:751-3.

4. International Association of Diabetes in Pregnancy Groups Consensus Panel. International association of diabetes and pregnancy study groups recommendations on the diagnosis and classi cation of hyperglycemia in pregnancy. Diabetes Care. 2010; 33: 676-82.

5. Classification and diagnosis of diabetes mellitus and other categories of glucose intolerance. National Diabetes Data Group. Diabetes. 1979; 28:1039-57.

6. Carpenter MW, Coustan DR. Criteria for screening tests for gestational diabetes. Am J Obstet Gynecol. 1982; 144:768-73.

7. Ferrara A, Hedderson MM, Quesenberry CP, Selby JV. Prevalence of gestational diabetes mellitus detected by the National Diabetes Data Group or the Carpenter and Coustan plasma glucose thresholds. Diabetes Care. 2002; 25:1625-30.

8. Ricart W, L pez J, Mozas J, Pericot A, Sancho MA, $G$ nzalez N, et al. Potential impact of American Diabetes Association (2000) criteria for diagnosis of gestational diabetes mellitus in Spain. Diabetologia. 2005; 48:1135-41.

9. Cheng YW, Block-Kurbisch I, Caughey AB. CarpenterCoustan criteria compared with the National Diabetes Data Group thresholds for gestational diabetes mellitus. Obstet Gynecol. 2009; 114:326-32.

10. Karcaaltincaba D, Kandemir O, Yalvac S, GuvendagGuven S, Haberal A. Prevalence of gestational diabetes mellitus and gestational impaired glucose tolerance in pregnant women evaluated by National Diabetes Data Group and Carpenter and Coustan criteria. Int J Gynecol Obstet. 2009; 106:246-9.

11. Chou CY, Lin CL, Yang CK, Yang WC, Lee FK, Tsai
MS. Pregnancy outcomes of Taiwanese women with gestational diabetes mellitus: a comparison of Carpenter-Coustan and National Diabetes Data Group criteria. J Womens Health (Larchmt). 2010; 19:935-9.

12. Berggren EK, Boggess KA, Stuebe AM, Jonsson Funk M. National Diabetes Data Group vs CarpenterCoustan criteria to diagnose gestational diabetes. Am J Obstet Gynecol. 2011; 205:253.e1-7.

13. Sunsaneevithayakul P, Boriboonhirunsarn D, Sutanthavibul A, Ruangvutilert P, Kanokpongsakdi S, Singkiratana D, et al. Risk factor-based selective screening program for gestational diabetes mellitus in Siriraj Hospital: result from clinical practice guideline. J Med Assoc Thai. 2003; 86:708-14.

14. Gokcel A, Bagis T, Killicadag EB, Tarim E, Guvener N. Comparison of the criteria for gestational diabetes mellitus by NDDG and Carpenter and Coustan, and the outcomes of pregnancy. J Endocrinol Invest. 2002; 25:357-61.

15. Ferrara A, Weiss NS, Hedderson MM, Quesenberry CP, Selby JV, Ergas IJ, et al. Pregnancy plasma glucose levels exceeding the American Diabetes Association thresholds, but below the National Diabetes Data Group thresholds for gestational diabetes mellitus, are related to the risk of neonatal macrosomia, hypoglycaemia, and hyperbilirubinaemia. Diabetologia. 2007; 50:298-306.

16. Boriboonhirunsarn D, Sunsaneevithayakul P. Abnormal results on a second testing and risk of gestational diabetes in women with normal baseline glucose levels. Int J Gynaecol Obstet. 2008; 100:147-53.

17. Boriboonhirunsarn D, Talungjit $P$, Sunsaneevithayakul P, Sirisomboon R. Adverse pregnancy outcomes in gestational diabetes mellitus. J Med Assoc Thai. 2006; 89:S23-8. 